\title{
Quantum theory of fibre Bragg grating solitons*
}

\author{
Ray-Kuang Lee ${ }^{1,2}$ and Yinchieh Lai ${ }^{1,3}$ \\ ${ }^{1}$ Institute of Electro-Optical Engineering, National Chiao-Tung University, Hsinchu, Taiwan, \\ R.O.C. 300 \\ ${ }^{2}$ National Center for High-Performance Computing, Hsinchu, Taiwan, R.O.C. 300 \\ E-mail: yclai@mail.nctu.edu.tw
}

Received 15 November 2003, accepted for publication 10 February 2004

Published 27 July 2004

Online at stacks.iop.org/JOptB/6/S638

doi:10.1088/1464-4266/6/8/003

\begin{abstract}
Following the pioneering work of Professor Haus, a general quantum theory for bi-directional nonlinear optical pulse propagation problems is developed and applied to study the quantum properties of fibre Bragg grating solitons. Fibre Bragg grating solitons are found to be automatically amplitude squeezed after passing through the grating and the squeezing ratio saturates after a certain grating length. The optimal squeezing ratio occurs when the pulse energy is slightly above the fundamental soliton energy. One can also compress the soliton pulsewidth and enhance the squeezing simultaneously by using an apodized grating, as long as the solitons evolve adiabatically.
\end{abstract}

Keywords: squeezed light, quantum solitons, photonic bandgap materials

(Some figures in this article are in colour only in the electronic version)

\section{Introduction}

Solitons are particle-like waves that propagate in dispersive or absorptive media without changing their pulse shapes and can survive after collisions. Various types of optical soliton phenomenon have been studied extensively in the area of nonlinear optical physics. These include the nonlinear Schrödinger solitons in dispersive optical fibres, spatial and vortex solitons in photorefractive materials/waveguides, and cavity solitons in resonators [1]. Solitons in optical fibres were first predicted by Hasegawa and Tappert [2] in 1973 and were first observed experimentally by Mollenauer et al [3] in 1980. Since then, the idea of using solitons for long-haul optical transmission has been an attractive research area with rapid progress [4]. In 1986, Gordon and Haus pointed out that the spontaneous emission from optical amplifiers in the optical link can cause the timing jitter variance of the solitons to be proportional to the cube of the propagation distance [5]. This effect, known as the Gordon-Haus effect, will place an upper limit on the achievable bit rate in long-haul soliton

\footnotetext{
* The authors would like to use this paper to express their deep thanks and respect to Professor Hermann A Haus for the inspiration they have directly or indirectly received from him.

3 Author to whom any correspondence should be addressed.
}

communication systems, even though it was found out later that one can overcome this limitation somewhat by using guiding filters $[6,7]$.

The spontaneous emission effects on soliton propagation already call for a rigorous quantum theory for soliton propagation, even though it may still be possible to treat them semi-classically. In 1987, Carter and Drummond et al $[8,9]$ used the positive- $P$ representation approach to transform the quantum nonlinear Schrödinger equation into stochastic nonlinear equations with noise terms. They then solved the stochastic nonlinear equations numerically and showed that solitons are quantum mechanically squeezed during propagation. The squeezing ratio of the quantum solitons was later calculated with the inclusion of the homodyne detection scheme [10]. Since then, the quantum theory of travelling-wave optical solitons has been intensively developed and several approaches have been successfully carried out. The studied soliton systems include the family of nonlinear Schrödinger solitons [9, 11-14] as well as the self-inducedtransparency solitons [15]. The quantum theory of nonlinear Schrödinger solitons has also been directly applied to study soliton communication problems [16-18].

The quantum theory of nonlinear Schrödinger solitons turns out to be an very interesting one. It was sometimes 
less well known that the quantum nonlinear Schrödinger equation is the evolution equation of a one-dimensional bosonic system with $\delta$-function interaction under the second quantization framework [19]. By solving the problem in the Schrödinger picture using the Bethe ansatz method, one can construct bound-state eigensolutions which are closely related to the soliton phenomenon. In 1989, Lai and Haus noticed this correspondence and constructed the exact soliton states by the Bethe ansatz method [12]. At the same time they also developed an approximate nonlinear analysis based on the time-dependent Hartree approximation [11]. In the subsequent year, a quantum soliton perturbation theory based on the linearization approach was successfully carried out by Haus and Lai [13]. The squeezing ratio of the soliton parts can be predicted by a simple analytic formulation and the optimal local oscillator pulse shape can be determined. This quantum soliton perturbation theory was later reformulated to make it clearer [17] and eventually led to the development of the back-propagation method [20], which is a general numerical method for calculating the quantum noises of nonlinear pulse propagation problems based on the linearization approximation. The aim of the present paper is to further pursue the theoretical development along this line by generalizing the theory of quantum solitons to bidirectional nonlinear pulse propagation problems and to study the quantum properties of Bragg grating solitons in particular.

On the side of experimental progress, many experiments for actually measuring the soliton squeezing have been carried out since 1990, with the advance of stable pulse lasers and high quantum efficiency detectors at optical communication wavelengths. To provide a stable experimental set-up against the environmental fluctuations, Shirasaki and Haus proposed to use a Sagnac loop in soliton squeezing experiments for measuring the quadrature squeezing [21]. The scheme is actually a squeezed vacuum state generator that can output squeezed vacuum states. Soliton squeezing from a Sagnac fibre interferometer was first observed by Rosenbluh and Shelby in 1991 (1.7 dB below the shot noise) [22]. Using the same set-up but at a different wavelength, Bergman and Haus also succeeded in observing squeezing with non-soliton pulses ( $4.9 \mathrm{~dB}$ below the shot noise) [23] in the same year. Since then, larger quadrature squeezing from fibres has been obtained with a gigahertz erbium-doped fibre laser to suppress the guided acoustic-wave Brillouin scattering (GAWBS) and a $6.1 \mathrm{~dB}$ noise reduction below the shot noise has been reported [24]. One can also produce amplitude squeezing by using solitons with an external spectral filter [25] or with an imbalance Sagnac loop interferometer [26]. Squeezing generation with an imbalance Sagnac loop interferometer in the normaldispersion region has also been demonstrated [27]. Recently, quantum correlation between different spectral components of the squeezed soliton has also been studied extensively in the literature [28]. The above brief description summarizes the exciting development that has been carried out for squeezing generation using optical fibres.

In recent years, with the advance of new fabrication technologies, it becomes more feasible to actually utilize one- or higher-dimensional periodic dielectric structures (or especially the photonic bandgap crystals) [29] to modify the properties of the spontaneous emission as well as the propagation of waves. The fibre Bragg gratings (FBGs) are one-dimensional periodic structures with weak index modulation. The simplest way to induce 1D Bragg gratings inside optical fibres is with side-illumination of the UV interference light. The FBG formed this way can be viewed as a 1D photonic bandgap crystal for the guiding mode of the single-mode fibre [30]. Interesting soliton phenomena known as fibre Bragg grating solitons [31, 32] can be found if the fibre Bragg grating has the third-order Kerr nonlinearity. The propagation of optical pulses inside the nonlinear FBG can be described by the nonlinear coupled mode equations (NCMEs). Intuitively, the fibre Bragg grating soliton is formed when the input pulse has suitable pulse-width and peak intensity such that the nonlinear Kerr effect is large enough to compensate the high anomalous dispersion near one of the bandedges of the FBG.

From the theoretical point of view, fibre Bragg grating solitons belong to the class of bi-directional pulse propagation problems, where the quantum theory still lacks enough consideration. Most of the previous studies on fibre Bragg grating solitons have been on the classical effects and there is almost no reported result on their quantum properties. It is the aim of this study to bridge this gap by developing a general quantum theory for bi-directional pulse propagation problems and particularly to apply the theory to study the case of fibre Bragg grating solitons. It will be shown that the fibre Bragg grating soliton pulses will quantum mechanically be amplitude squeezed after passing through the fibre grating and the squeezing ratio will be calculated theoretically. We use the linearization approach to study the quantum effects of FBG solitons by extending the backpropagation method previously developed [20] to the case of nonlinear bi-directional propagation problems. By following the same spirit of the back-propagation method, we will first derive a set of linear adjoint equations from the linearized nonlinear coupled mode equations in such a way that any inner product between the solutions of the two equation sets is conserved during the time evolution. In this way, the variance of the measured operator as well as its squeezing ratio can be calculated readily for a given measurement characteristic function. The squeezing ratio of FBG solitons will be found to exhibit interesting relations with the fibre grating length as well as with the intensity of the input pulse.

In contrast to uniform fibre gratings, nonuniform fibre gratings with chirp and/or apodization have shown some potential for pulse compression applications [33]. The theory and experiment for Bragg grating solitons propagated in apodized FBGs have also been developed and demonstrated [34, 35]. Some new results on slowing down FBG solitons in apodized fibre gratings have also been carried out [36]. In the present paper the quantum fluctuations of FBG solitons in nonuniform fibre gratings will also be studied. We find that one can compress the soliton pulsewidth and enhance the squeezing simultaneously by using an apodized grating, as long as the solitons evolve adiabatically.

The paper is organized as follows: in section 2 the model of the nonlinear coupled mode equations for bi-directional wave propagation in a uniform FBG is reviewed. In section 3 we use the back-propagation method to calculate the quantum fluctuations of FBG solitons and the characteristics of the 


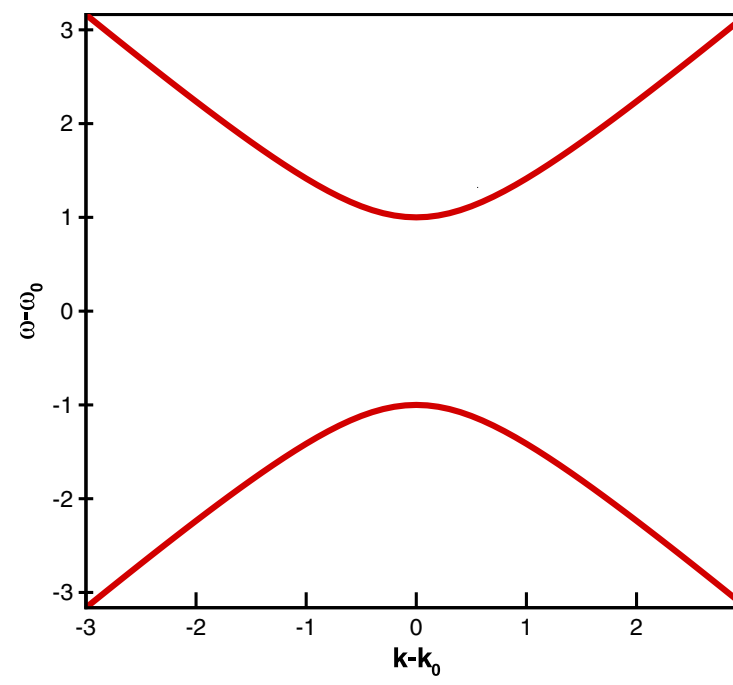

Figure 1. Dispersion relation of linear fibre Bragg gratings.

squeezing ratio are discussed. In section 4 the quantum fluctuations of FBG solitons in nonuniform fibre Bragg gratings are studied. Finally, a brief conclusion is given in section 5 .

\section{Fibre Bragg grating solitons}

We start the derivation by considering the linear wave propagation problem in a one-dimensional periodic structure:

$$
\frac{\partial^{2} E}{\partial z^{2}}-\frac{n^{2}(z)}{c^{2}} \frac{\partial^{2} E}{\partial t^{2}}=0
$$

Here the dielectric constant $n^{2}(z)=\bar{n}^{2}+\tilde{\epsilon}(z)$ is a periodic function of the propagation distance, with the spatial average refractive index $\bar{n}$ and the period $\Lambda$. We are interested in the light field at a frequency near the Bragg condition $\omega_{0}=k_{0} c / \bar{n}$, where $c$ is the light velocity in free space, and $k_{0}=\pi / \Lambda$. For FBGs, we can expand the periodic index perturbation function $\tilde{\epsilon}(z)$ by the Fourier series and only keep the phase-matching \pm 1 order terms, i.e. $\tilde{\epsilon}(z)=$ $2 \tilde{\epsilon}_{0} \cos \left(2 k_{0} z\right)$. One then decomposes the light field into the forward $\left(U_{a}\right)$ and backward $\left(U_{b}\right)$ propagation pulses, $E(z, t)=$ $U_{a}(z, t) \mathrm{e}^{-\mathrm{i}\left(\omega t-k_{0} z\right)}+U_{b}(z, t) \mathrm{e}^{-\mathrm{i}\left(\omega t+k_{0} z\right)}+$ c.c., and obtains the following linear coupled mode equations:

$$
\begin{aligned}
& \frac{1}{v_{\mathrm{g}}} \frac{\partial}{\partial t} U_{a}(z, t)+\frac{\partial}{\partial z} U_{a}=\mathrm{i} \delta U_{a}+\mathrm{i} \kappa U_{b} \\
& \frac{1}{v_{\mathrm{g}}} \frac{\partial}{\partial t} U_{b}(z, t)-\frac{\partial}{\partial z} U_{b}=\mathrm{i} \delta U_{b}+\mathrm{i} \kappa U_{a}
\end{aligned}
$$

where $v_{\mathrm{g}}=\bar{n} / c$ is the group velocity of the pulses, $\delta=\omega-\omega_{0}$ is the wavelength detuning parameter, and $\kappa=\omega_{0} \tilde{\epsilon} / 2 \bar{n} c$ is the coupling coefficient. In figure 1 one can see that the dispersion relation of this set of coupled-mode equations has a bandgap at the frequency (wavelength) that satisfies the Bragg condition $k=k_{0}$.

If the third order nonlinearity of the optical fibre needs to be taken into account, one can model the problem by using the above coupled mode equations that describe the coupling between the forward and the backward propagating waves in a uniform FBG with the addition of the self-phase and crossphase modulation effects. With the nonlinear terms, this set of NCMEs has analytical soliton solutions for the case of infinite grating length, as is shown by Aceves and Wabnitz [31] with the introduction of the massive Thirring model. However, for nonlinear FBGs of finite length, no analytic solution can be found. So in our studies we directly use the finite difference numerical simulation method with the parameters based on the first experiment reported in the literature [32]. We consider a $60 \mathrm{ps}$ full width at half maximum (FWHM) sech-shaped pulse incident into a uniform grating with $15.0 \mathrm{~cm}^{-1}$ wavenumber detuning from the centre of the bandgap. The coupling strength of the fibre grating is $10 \mathrm{~cm}^{-1}$, the nonlinear coefficient $\Gamma$ is $0.018 \mathrm{~cm} \mathrm{GW}^{-1}$, and the group velocity $v_{\mathrm{g}}$ is chosen to be $c / n$ with $n=1.5$ and $c$ being the speed of light in free space. When the input peak intensity is below the required value for forming a solitary pulse in the FBG (about $4.5 \mathrm{GW} \mathrm{cm}^{-2}$ in this case), the peak intensity of the pulse will decrease along the propagation. On the other hand, when the input peak intensity is above $4.5 \mathrm{GW} \mathrm{cm}^{-2}$, the peak intensity of the pulse oscillates during the propagation within the grating. Only when the nonlinearity can exactly compensate the dispersion induced by the FBG can one have a stable solitary pulse inside the grating.

\section{Quantum fibre Bragg grating solitons}

After finding these classical solutions, we now turn to the calculation of the quantum properties. The Hamiltonian of the system can be expressed by the forward $\left(\hat{U}_{a}\right)$ and backward $\left(\hat{U}_{b}\right)$ field operators as follows [37]:

$$
\begin{aligned}
\mathcal{H}= & -v_{\mathrm{g}}\left\{\delta \int \mathrm{d} z\left(\hat{U}_{a}^{\dagger} \hat{U}_{a}+\hat{U}_{b}^{\dagger} \hat{U}_{b}\right)\right. \\
& +\mathrm{i} \int \mathrm{d} z\left(\hat{U}_{a}^{\dagger} \frac{\partial}{\partial z} \hat{U}_{a}-\hat{U}_{b}^{\dagger} \frac{\partial}{\partial z} \hat{U}_{b}\right) \\
& +\kappa \int \mathrm{d} z\left(\hat{U}_{a}^{\dagger} \hat{U}_{b}+\hat{U}_{b}^{\dagger} \hat{U}_{a}\right) \\
& +\frac{\Gamma}{2} \int \mathrm{d} z\left(\hat{U}_{a}^{\dagger} \hat{U}_{a}^{\dagger} \hat{U}_{a} \hat{U}_{a}+\hat{U}_{b}^{\dagger} \hat{U}_{b}^{\dagger} \hat{U}_{b} \hat{U}_{b}\right) \\
& \left.+\Gamma \int \mathrm{d} z\left(\hat{U}_{a}^{\dagger} \hat{U}_{b}^{\dagger} \hat{U}_{b} \hat{U}_{a}+\hat{U}_{b}^{\dagger} \hat{U}_{a}^{\dagger} \hat{U}_{a} \hat{U}_{b}\right)\right\} .
\end{aligned}
$$

Here the field operators $\hat{U}_{a}$ and $\hat{U}_{b}$ must obey the usual equal time bosonic commutation relations.

$$
\begin{gathered}
{\left[\hat{U}_{a}\left(z_{1}, t\right), \hat{U}_{a}^{\dagger}\left(z_{2}, t\right)\right]=\delta\left(z_{1}-z_{2}\right)} \\
{\left[\hat{U}_{b}\left(z_{1}, t\right), \hat{U}_{b}^{\dagger}\left(z_{2}, t\right)\right]=\delta\left(z_{1}-z_{2}\right)} \\
{\left[\hat{U}_{a}\left(z_{1}, t\right), \hat{U}_{a}\left(z_{2}, t\right)\right]=\left[\hat{U}_{a}^{\dagger}\left(z_{1}, t\right), \hat{U}_{a}^{\dagger}\left(z_{2}, t\right)\right]=0} \\
{\left[\hat{U}_{b}\left(z_{1}, t\right), \hat{U}_{b}\left(z_{2}, t\right)\right]=\left[\hat{U}_{b}^{\dagger}\left(z_{1}, t\right), \hat{U}_{b}^{\dagger}\left(z_{2}, t\right)\right]=0} \\
{\left[\hat{U}_{a}\left(z_{1}, t\right), \hat{U}_{b}\left(z_{2}, t\right)\right]=\left[\hat{U}_{a}\left(z_{1}, t\right), \hat{U}_{b}^{\dagger}\left(z_{2}, t\right)\right]=0 .}
\end{gathered}
$$

In the Heisenberg picture we can derive the quantum nonlinear coupled mode equations (QNCMEs) by the 
Hamiltonian in equation (4)

$$
\begin{aligned}
& \frac{1}{v_{\mathrm{g}}} \frac{\partial}{\partial t} \hat{U}_{a}(z, t)+\frac{\partial}{\partial z} \hat{U}_{a}=\mathrm{i} \delta \hat{U}_{a} \\
& \quad+\mathrm{i} \kappa \hat{U}_{b}+\mathrm{i} \Gamma \hat{U}_{a}^{\dagger} \hat{U}_{a} \hat{U}_{a}+2 \mathrm{i} \Gamma \hat{U}_{b}^{\dagger} \hat{U}_{b} \hat{U}_{a} \\
& \frac{1}{v_{\mathrm{g}}} \frac{\partial}{\partial t} \hat{U}_{b}(z, t)-\frac{\partial}{\partial z} \hat{U}_{b}=\mathrm{i} \delta \hat{U}_{b}+\mathrm{i} \kappa \hat{U}_{a} \\
& \quad+\mathrm{i} \Gamma \hat{U}_{b}^{\dagger} \hat{U}_{b} \hat{U}_{b}+2 \mathrm{i} \Gamma \hat{U}_{a}^{\dagger} \hat{U}_{a} \hat{U}_{b} .
\end{aligned}
$$

This derivation automatically proves that the QNCMEs preserve the commutation brackets. The form of QNCMEs in equations (5) and (6) is the same as the classical NCMEs, except that it is now a set of quantum operator equations. For optical solitons with a large average photon number, we can safely use the linearization approximation to study their quantum effects. By setting

$$
\begin{aligned}
& \hat{U}_{a}(z, t)=U_{a 0}(z, t)+\hat{u}_{a}(z, t) \\
& \hat{U}_{b}(z, t)=U_{b 0}(z, t)+\hat{u}_{b}(z, t)
\end{aligned}
$$

where $U_{a 0}$ and $U_{b 0}$ are classical fibre Bragg grating soliton solutions of NCMEs, one can obtain a set of linear quantum operator equations that describe the evolution of the quantum fluctuations associated with the fibre Bragg grating solitons after the linearization approximation is performed.

$$
\begin{aligned}
& \frac{1}{v_{\mathrm{g}}} \frac{\partial}{\partial t}\left(\begin{array}{c}
\hat{u}_{a} \\
\hat{u}_{b}
\end{array}\right)=\left(\begin{array}{c}
-\frac{\partial}{\partial z}+\mathrm{i} \delta+2 \mathrm{i} \Gamma\left|U_{a 0}\right|^{2}+2 \mathrm{i} \Gamma\left|U_{b 0}\right|^{2} \\
\mathrm{i} \kappa+2 \mathrm{i} \Gamma U_{a 0}^{*} U_{b 0}
\end{array}\right. \\
& \left.\begin{array}{c}
\mathrm{i} \kappa+2 \mathrm{i} \Gamma U_{a 0} U_{b 0}^{*} \\
+2 \mathrm{i} \Gamma\left|U_{a 0}\right|^{2}+2 \mathrm{i} \Gamma\left|U_{b 0}\right|^{2}
\end{array}\right)\left(\begin{array}{l}
\hat{u}_{a} \\
\hat{u}_{b}
\end{array}\right) \\
& +\left(\begin{array}{cc}
\mathrm{i} \Gamma U_{a 0}^{2} & 2 \mathrm{i} \Gamma U_{a 0} U_{b 0} \\
2 \mathrm{i} \Gamma U_{a 0} U_{b 0} & \mathrm{i} \Gamma U_{b 0}^{2}
\end{array}\right)\left(\begin{array}{c}
\hat{u}_{a}^{\dagger} \\
\hat{u}_{b}^{\dagger}
\end{array}\right) .
\end{aligned}
$$

This set of equations is still a set of quantum operator equations for the quantum perturbation field operators $\hat{u}_{a}(z, t)$ and $\hat{u}_{b}(z, t)$, which have to satisfy the same equal time commutation relations as the unperturbed field operators $\hat{U}_{a}(z, t)$ and $\hat{U}_{b}(z, t)$.

To solve the linear quantum operator equations in equation (7) by the back-propagation method, we define the inner product operation according to

$$
\langle\vec{f} \mid \overrightarrow{\hat{g}}\rangle=\frac{1}{2} \int \mathrm{d} z\left[f_{a}^{*} \hat{g}_{a}+f_{a} \hat{g}_{a}^{\dagger}+f_{b}^{*} \hat{g}_{b}+f_{b} \hat{g}_{b}^{\dagger}\right]
$$

We then seek a set of adjoint equations which satisfies the following property:

$$
\frac{\mathrm{d}}{\mathrm{d} t}\left\langle\vec{u}^{\mathrm{A}} \mid \overrightarrow{\hat{u}}\right\rangle=0
$$

In other words, the inner product between the solutions of the two equation sets is preserved along the time axis. With this desired property, we can express the inner product of the output quantum perturbation operator with a projection function in terms of the input quantum field operators which have the known quantum characteristics. This will allow us to calculate the quantum fluctuations of any inner product between the output quantum operator and a given projection function. It

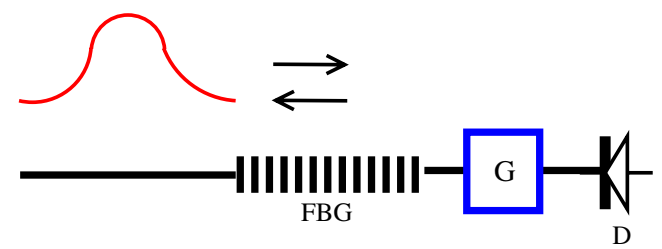

Figure 2. Measurement scheme of direct detection for observing FBG soliton squeezing. Here $\mathrm{G}$ is a gating device which will block out all the transmittive pulses but the first one; D is an optical detector.

is not difficult to show that the set of adjoint equations for the perturbed QNCMES in equation (7) is given by

$$
\begin{gathered}
\frac{1}{v_{\mathrm{g}}} \frac{\partial}{\partial t}\left(\begin{array}{c}
u_{a}^{\mathrm{A}} \\
u_{b}^{\mathrm{A}}
\end{array}\right)=\left(\begin{array}{c}
-\frac{\partial}{\partial z}+\mathrm{i} \delta+2 \mathrm{i} \Gamma\left|U_{a 0}\right|^{2}+2 \mathrm{i} \Gamma\left|U_{b 0}\right|^{2} \\
\mathrm{i} \kappa+2 \mathrm{i} \Gamma U_{a 0}^{*} U_{b 0} \\
\mathrm{i} \kappa+2 \mathrm{i} \Gamma U_{a 0} U_{b 0}^{*} \\
\frac{\partial}{\partial z}+\mathrm{i} \delta+2 \mathrm{i} \Gamma\left|U_{a 0}\right|^{2}+2 \mathrm{i} \Gamma\left|U_{b 0}\right|^{2}
\end{array}\right)\left(\begin{array}{c}
u_{a}^{\mathrm{A}} \\
u_{b}^{\mathrm{A}}
\end{array}\right) \\
+\left(\begin{array}{cc}
-\mathrm{i} \Gamma U_{a 0}^{2} & -2 \mathrm{i} \Gamma U_{a 0} U_{b 0} \\
-2 \mathrm{i} \Gamma U_{a 0} U_{b 0} & -\mathrm{i} \Gamma U_{b 0}^{2}
\end{array}\right)\left(\begin{array}{c}
u_{a}^{\mathrm{A} *} \\
u_{b}^{\mathrm{A} *}
\end{array}\right) .
\end{gathered}
$$

Actually, under the linearization approximation the measurement of a physical quantity can always be expressed as an inner product between a measurement characteristic function and the perturbed quantum field operator [20]. By back-propagating the classical adjoint equation set with the measurement characteristic function as the initial condition, one can express the measured inner product as an inner product between the input quantum operator and the back-propagated measurement function. It is then quite easy to calculate the quantum uncertainties of the measured quantity if the quantum characteristics of the input quantum field operators are known. Typically we will assume the input quantum state to be a coherent state. The squeezing ratio of the measured quantity is defined according to

$$
\begin{aligned}
R(T) & =\frac{\operatorname{var}[\langle\vec{f}(z)| \overrightarrow{\hat{u}}(z, t=T)]}{\operatorname{var}[\langle\vec{f}(z)| \overrightarrow{\hat{u}}(z, t=0)]} \\
& =\frac{\operatorname{var}\left[\left\langle\vec{F}_{T}(z)\right| \overrightarrow{\hat{u}}(z, t=0)\right]}{\operatorname{var}[\langle\vec{f}(z)| \overrightarrow{\hat{u}}(z, t=0)]} .
\end{aligned}
$$

Here $\operatorname{var}[\cdot]$ means the variance, $\vec{f}(z)$ is the original projection function and $\vec{F}_{T}(z)$ is the back-propagated projection function. The choice of the characteristic function $\vec{f}(z)=\left(f_{a}(z), f_{b}(z)\right)^{T}$ will depend on the measurement to be performed. When the measured quantity is the photon number of the pulse, the characteristic function $f_{a}(z)$ is simply the normalized classical output pulse [13] and $f_{b}(z)=0$. If the homodyne detection is used, then $f_{a}(z)$ is the normalized local oscillator pulse.

In figure 2 we illustrate the possible direct detection scheme for measuring the photon number squeezing of the FBG solitons. To avoid the complication due to the multiple transmitted pulses of the FBG, it may be necessary to use a time-gating device to make sure that only the first transmitted pulse is detected.

Based on the formulation given above, we now apply the developed quantum theory to the case of a pulse incident into a uniform nonlinear FBG and calculate the photon number fluctuations of the first transmitted pulse. First of all, 

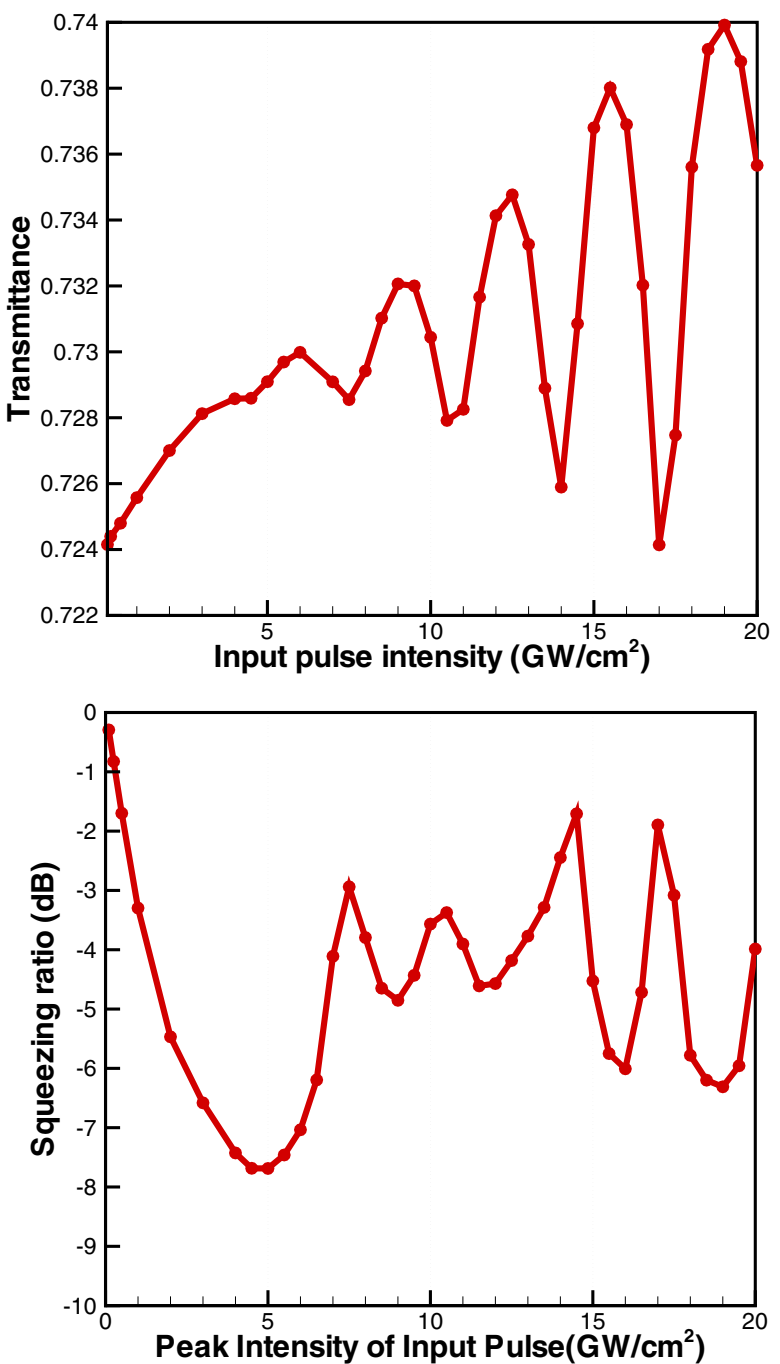

Figure 3. Transmittance (top) and photon number squeezing ratio (bottom) for fibre Bragg grating solitons with different input intensities.

the transmittance of a $50 \mathrm{~cm}$ long $\mathrm{FBG}$ versus different intensities of the input pulse is plotted in the top curve of figure 3. It can be seen that the transmittance of the FBG is a nonlinear oscillating function of the input pulse intensity. The calculated photon number squeezing ratio is shown at the bottom of figure 3 with the same parameters. One can see that the squeezing ratio decreases monotonically when the input intensity is below the intensity of the fundamental soliton. The optimum squeezing ratio occurs when the pulse energy of the soliton is slightly larger than that of the fundamental soliton. The curve begins to oscillate strongly when the intensity is much larger than that of the fundamental soliton. One important observation is that the oscillation behaviour of the FBG transmittance and the squeezing ratio matches very well. That is, the squeezing ratio has a local minimum when the transmission has a local maximum. This agrees with the intuitive expectation that larger amplitude squeezing should occur when the transmittance curve is saturated. Another way to intuitively understand the results is as follows. The periodic grating structure acts like a spectral filter which can filter out the noisier high frequency components in the

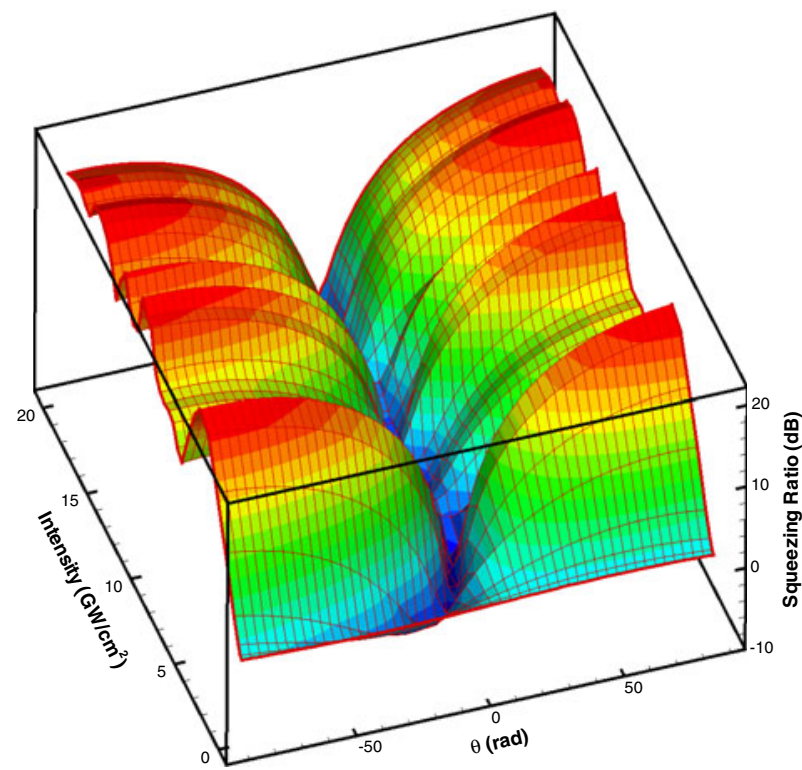

Figure 4. Squeezing ratio of the quadrature field versus the input intensity and the local oscillator phase.

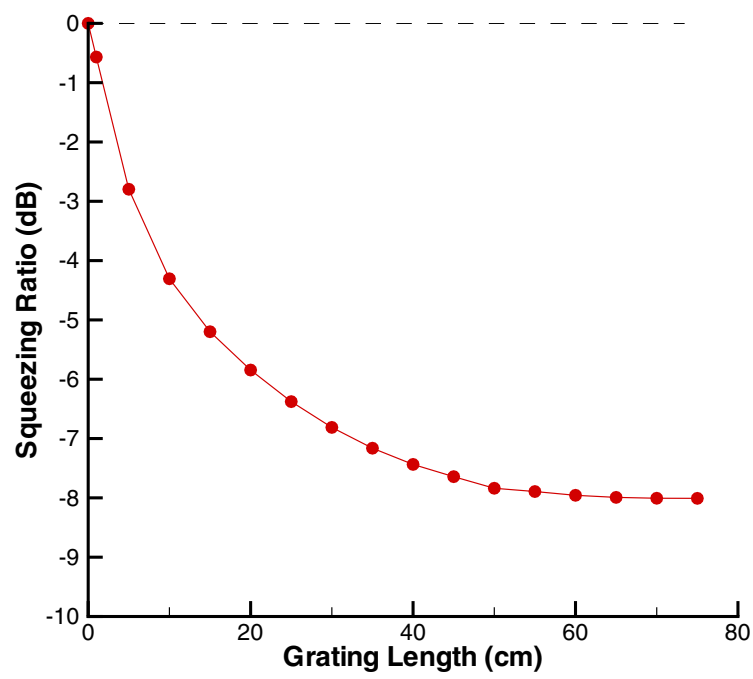

Figure 5. Photon number squeezing ratio for Bragg solitons after propagating through different lengths of FBGs.

soliton spectrum and produce a net photon number (amplitude) squeezing effect just as in the previous soliton amplitude squeezing experiments where a spectral filter is cascaded after a nonlinear fibre $[25,38]$.

To make sure that the FBG solitons are actually amplitude squeezed, we perform another calculation to simulate the squeezing ratio when the homodyne detection scheme is used and assume that the local oscillator pulse is exactly the classical output pulses. With the homodyne detection scheme, one has the additional degree of freedom to adjust the relative phase between the local oscillator and the signal. Figure 4 plots the squeezing ratio for different input intensities and for different local oscillator phases when propagating through a constant FBG length $\left(50 \mathrm{~cm}^{2}\right)$. One can see that for small input intensities (below $5 \mathrm{GW} \mathrm{cm}^{-2}$ ) the squeezing direction is close to but not exactly in the photon number (or amplitude) quadrature, $\theta=0$. However, when the intensity of the FBG 


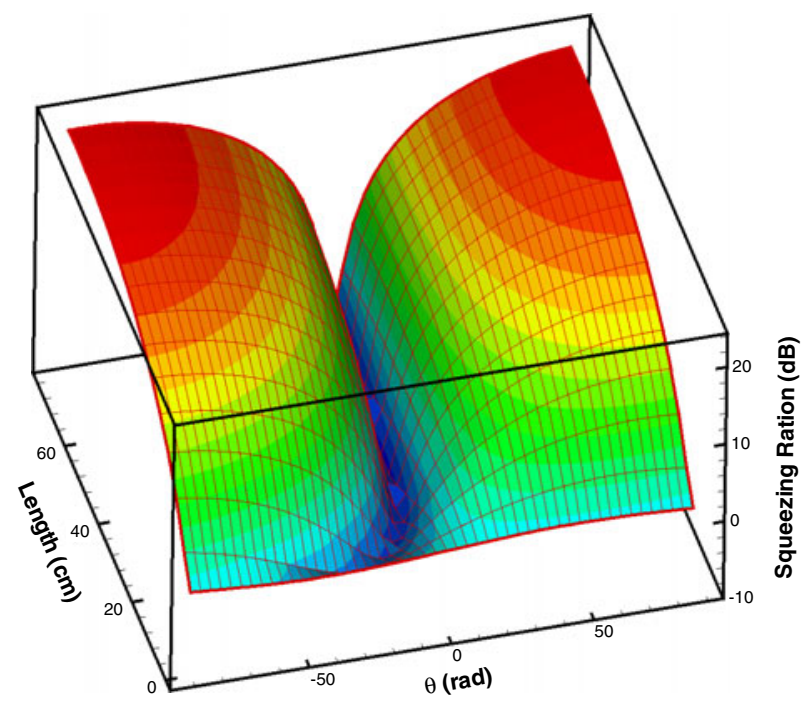

Figure 6. Squeezing ratio of the quadrature field versus the FBG length and the local oscillator phase.

soliton is large enough, the squeezing direction will be in the photon number quadrature.

Figure 5 shows the dependence of the photon number squeezing ratios for different FBG lengths with a constant input intensity $\left(I=4.5 \mathrm{GW} \mathrm{cm}^{-2}\right)$. We find that the squeezing ratio monotonically decreases with increasing FBG length and saturates at a length around $60 \mathrm{~cm}$. Intuitively, the filtering effects of the FBG will unavoidably introduce additional noise on the light fields and will eventually cause the squeezing ratio to become saturated. In figure 6 we also plot the squeezing ratio for different FBG lengths and for different local oscillator phases with a constant input intensity $\left(I=4.5 \mathrm{GW} \mathrm{cm}^{-2}\right)$. Again when the FBG length is long enough, the squeezing direction will be in the in-phase (photon number) quadrature.

\section{Quantum FBG solitons in nonuniform gratings}

It is well known that one can engineer the dispersion along the FBG by using a nonuniform FBG. Such apodized nonlinear FBGs have been used for adiabatic soliton pulse compression within a very short length of several centimetres [34, 35]. Intuitively, the solitons with higher peak intensities will exhibit large squeezing due to higher nonlinear effects. It is thus expected that one should be able to compress the pulsewidth of the FBG soliton and enhance its squeezing ratio simultaneously with the use of a suitably apodized FBG. To verify this idea, here we consider a nonuniform FBG which has a position dependent coupling coefficient described by

$$
\kappa(z)=\kappa_{0}+\alpha z
$$

where $\kappa_{0}=\omega_{0} \tilde{\epsilon} / 2 \bar{n} c$ is the initial coupling coefficient and $\alpha$ is the slope of the coupling coefficient.

In the following calculation we consider the same $60 \mathrm{ps}$ FWHM sech-shaped input pulse with the peak intensity of $I=$ $4.5 \mathrm{GW} \mathrm{cm}^{-2}$ for the nonuniform grating without changing any parameter. In figure 7 we plot the squeezing ratio versus the FBG length with a constant input intensity $\left(I=4.5 \mathrm{GW} \mathrm{cm}^{-2}\right)$ and different apodization slopes. The three curves in figure 7 correspond to different slopes of $\kappa(z)(\alpha=+0.04,0,-0.04)$

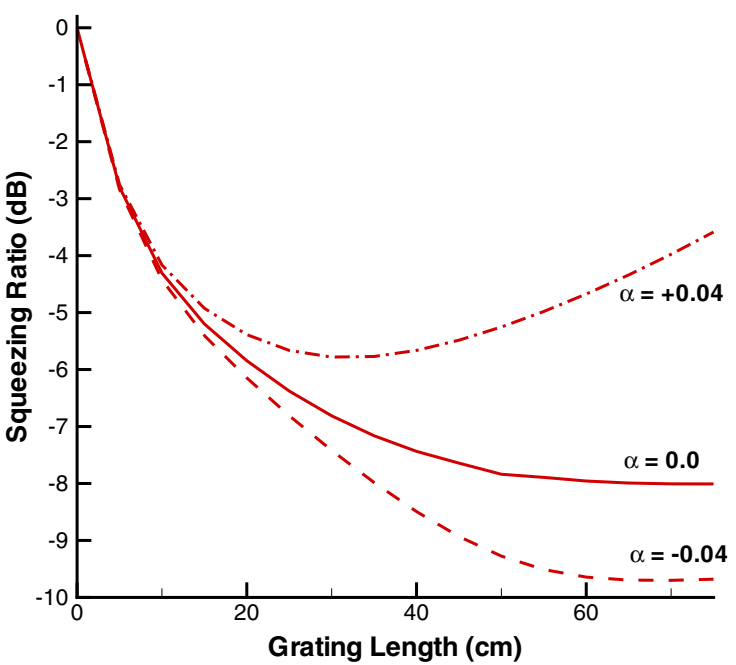

Figure 7. Photon number squeezing ratio versus the FBG length with a constant input intensity $\left(I=4.5 \mathrm{GW} \mathrm{cm}^{-2}\right)$. The coupling coefficient of the FBG is a function of propagating length: $\kappa(z)=\kappa_{0}+\alpha z$. The three curves correspond to different slopes of $\kappa(z)$ : solid curve, $\alpha=0$; dashed curve, $\alpha=-0.04$; dash-dotted curve, $\alpha=+0.04$.

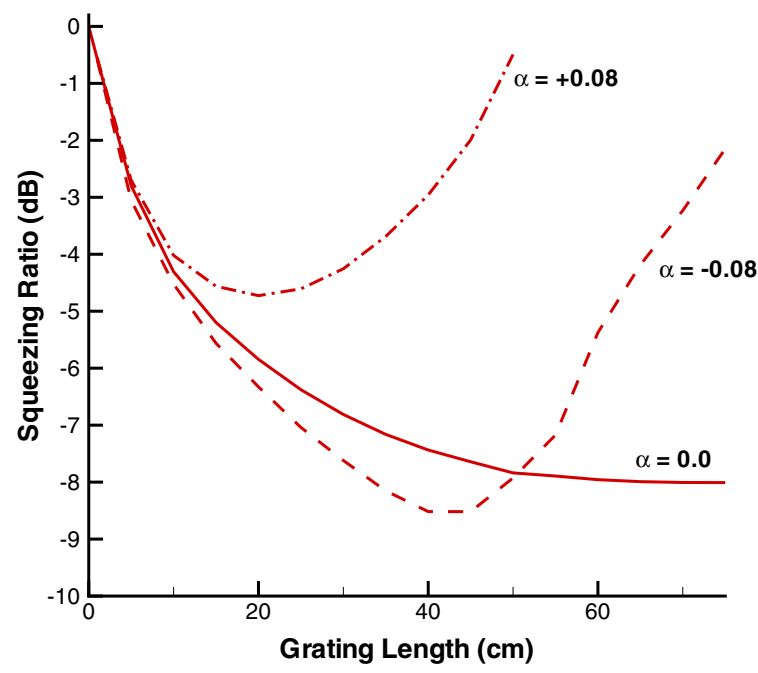

Figure 8. Photon number squeezing ratio versus the FBG length with different slopes of $\kappa(z)$ : solid curve, $\alpha=0$; dashed curve, $\alpha=-0.08$; dash-dotted curve, $\alpha=+0.08$. Other parameters are the same as those in figure 7.

respectively. When $\alpha=0.04 \mathrm{~cm}^{-2}$, the FWHM of the original FBG soliton (60 ps) will be adiabatically compressed to $30 \mathrm{ps}$ after propagating through a $70 \mathrm{~cm}$ FBG. Because of this, the achievable optimal squeezing ratio increases from 8 to $9.7 \mathrm{~dB}$ for the propagation distance of $70 \mathrm{~cm}$. On the other hand, if the slope of the coupling coefficient is positive, we have a broadened FBG soliton and the optimal squeezing ratio will be degraded. If the slope of the coupling coefficient is too large, then the soliton cannot be compressed adiabatically and thus the optimal squeezing ratio will eventually be degraded. This can be seen in figure 8 , where we plot the squeezing ratio versus the grating length with larger slopes $(\alpha=-0.08,+0.08)$. The result for the uniform FBG case $(\alpha=0)$ is also plotted for comparison. These results show that one can actually tailor the squeezing ratio of the FBG solitons as long as the soliton pulses evolve adiabatically. Recently, some new theoretical results for 
the dynamics of classical gap solitons in an apodized grating have been carried out [36]. It was shown that the velocity of the gap soliton can be essentially slowed down in the apodized grating and interesting soliton collision behaviours including the merge of the solitons were predicted. We shall investigate more about the other quantum dynamics of FBG solitons in apodized fibre gratings in the future.

\section{Conclusion}

In conclusion, by following the line of theoretical development on the quantum theory of optical solitons initiated by Professor Haus, we have developed a general quantum theory for bidirectional nonlinear optical pulse propagation problems and have especially used it to study the squeezing phenomena of fibre Bragg grating solitons. It has been shown for the first time that the output FBG soliton pulses will be amplitude squeezed automatically. The squeezing ratio of the FBG solitons exhibits an interesting relation with the fibre grating length as well as with the intensity of the input pulse. The squeezing ratio saturates after a certain grating length and the optimal squeezing ratio occurs when the intensity of the FBG soliton is slightly larger than that of the fundamental soliton. With the use of nonuniform FBGs, we also find that one can compress the FBG soliton and enhance its squeezing ratio simultaneously, as long as the soliton pulses evolve adiabatically. To actually measure the quantum fluctuations of the fibre Bragg grating solitons experimentally, we propose to use a time-gating device to block out other smaller multiple transmitted pulses and only directly detect the first transmitted pulse from the grating. Since our calculation is already based on the existing experimental parameters, it will be very interesting to see whether one can actually verify the theoretical predictions experimentally.

\section{Acknowledgments}

This work was supported in part by the National Science Council of the Republic of China under Grant No. NSC 932752-E-009-009-PAE, as well as by the Center for NanoScience and Technology in the United System of Taiwan.

\section{References}

[1] Kivshar Yu S and Agrawal G P 2003 Optical Solitons: From Fibers to Photonic Crystals (San Diego, CA: Academic)

[2] Hasegawa A and Tappert F 1973 Appl. Phys. Lett. 23142
[3] Mollenauer L F, Stolen R H and Gordon J P 1980 Phys. Rev. Lett. 451095

[4] Iannone E, Mecozzi A and Settembre M 1998 Nonlinear Optical Communication Networks (New York: Wiley-Interscience)

[5] Gordon J P and Haus H A 1986 Opt. Lett. 11665

[6] Mecozzi A, Moores J D, Haus H A and Lai Y 1991 Opt. Lett. 161841

[7] Kodama Y and Hasegawa A 1992 Opt. Lett. 1731

[8] Carter S J, Drummond P D, Reid M D and Shelby R M 1987 Phys. Rev. Lett. 581841

[9] Drummond P D and Carter S J 1987 J. Opt. Soc. Am. B 41565

[10] Carter S J, Drummond P D and Shelby R M 1989 Opt. Lett. 14 373

[11] Lai Y and Haus H A 1989 Phys. Rev. A 40844

[12] Lai Y and Haus H A 1989 Phys. Rev. A 40854

[13] Haus H A and Lai Y 1990 J. Opt. Soc. Am. B 7386

[14] Schmidt E, Knöll L, Welsch D-G, Zielonka M, König F and Sizmann A 2000 Phys. Rev. Lett. 853801

[15] Lai Y and Haus H A 1990 Phys. Rev. A 422925

[16] Haus H A 1991 J. Opt. Soc. Am. B 81122

[17] Lai Y 1993 J. Opt. Soc. Am. B 10475

[18] Lai Y 1993 IEEE J. Lightwave Technol. 11462

[19] Huang K 1987 Statistical Mechanics 2nd edn (New York: Wiley)

[20] Lai Y and Yu S-S 1995 Phys. Rev. A 51817

[21] Shirasaki M and Haus H A 1990 J. Opt. Soc. Am. B 730

[22] Rosenbluh M and Shelby R 1991 Phys. Rev. Lett. 66153

[23] Bergman K and Haus H A 1991 Opt. Lett. 16663

[24] Yu C X, Haus H A and Ippen E P 2001 Opt. Lett. 26669

[25] Friberg S R, Machida S, Werner M J, Levanon A and Mukai T 1996 Phys. Rev. Lett. 773775

[26] Krylov D and Bergman K 1998 Opt. Lett. 231390

[27] Krylov D, Bergman K and Lai Y 1999 Opt. Lett. 24774

[28] Spälter S, Korolkova N, König F, Sizmann A and Leuchs G 1998 Phys. Rev. Lett. 81786

[29] Joannopoulos J D, Meade R D and Winn J N 1995 Photonic Crystals: Molding the Flow of Light (Princeton, NJ: Princeton University Press)

[30] Erdogan T 1997 IEEE J. Lightwave Technol. 151277

[31] Aceves A B and Wabnitz S 1989 Phys. Lett. A 14137

[32] Eggleton B J, Slusher R E, de Sterke C M, Krug P A and Sipe J E 1996 Phys. Rev. Lett. 761627

[33] Eggleton B J, Lenz G and Litchinitser N M 2000 Fiber Integr. Opt. 19383

[34] Lenz G and Eggleton B J 1998 J. Opt. Soc. Am. B 152979

[35] Eggleton B J, de Sterke C M and Slusher R E 1999 J. Opt. Soc. Am. B 16587

[36] Mak W C K, Malomed B A and Chu P L 2003 Phys. Rev. E 68 026609

[37] Cheng Z and Kurizki G 1996 Phys. Rev. A 543576

[38] Opatrný T, Korolkova N and Leuchs G 2002 Phys. Rev. A 66 053813 